\section{Construyendo puentes entre investigación y políticas para la extensión de la protección social en salud en América Latina y el Caribe: una estrategia de cooperación conjunta}

\author{
Building bridges between research and policy to \\ extend social protection in health in Latin America \\ and the Caribbean: a strategy for cooperation
}

\author{
${ }^{1}$ Centro Internacional de \\ Investigaciones para el \\ Desarrollo-Oficina Regional, \\ Montevideo, Uruguay. \\ ${ }^{2}$ Health Policies \& Systems \\ Unit, Health Systems \\ Strengthening Area, \\ Pan American Health \\ Organization, \\ Washington DC, U.S.A. \\ ${ }^{3}$ Governance, Equity \\ and Health Program \\ Initiative, International \\ Development Research \\ Centre, Ottawa, Canada. \\ Correspondencia \\ R. Bazzani \\ Centro Internacional de \\ Investigaciones para el \\ Desarrollo-Oficina Regional. \\ Av. Brasil 2655, 11300 \\ Montevideo, Uruguay \\ rbazzani@idrc.org.uy
}

\begin{abstract}
The Pan American Health Organization (PAHO) and International Development Research Centre (IDRC) have promoted a joint initiative to design, implement, and evaluate innovative strategies for the Extension of Social Protection in Health $(S P H)$ in Latin America and the Caribbean (LAC), involving active partnership between researchers and research users. This initiative was based on a previous review of research on health sector reforms and the recommendations of the workshop on "Health Sector Reforms in the Americas: Strengthening the Links between Research and Policy" (Montreal, Canada, 2001). In its first phase, the initiative supported the development of proposals aiming to extend SPH, elaborated jointly by researchers and decision-makers. In the second phase, the implementation of five of these proposals was supported in order to promote the development of new SPH strategies and new stakeholder interaction models. In this edition of the journal, the process of linking researchers and decision-makers will be analyzed in the context of the five projects supported by this initiative.
\end{abstract}

Health Policy; Health Systems; Health Services
Roberto Bazzani 1

Eduardo Levcovitz 2

Soledad Urrutia 1

Christina Zarowsky ${ }^{3}$

\section{Antecedentes}

El Centro Internacional de Investigaciones para el Desarrollo de Canadá (IDRC) y la Organización Panamericana de la Salud (OPS) han promovido en los últimas años una estrategia de cooperación conjunta en el campo de la investigación en políticas y sistemas de salud, con especial énfasis en la articulación de esfuerzos entre académicos y tomadores de decisión, y con foco de atención en el análisis de las reformas y los desafíos actuales de las políticas y sistemas de salud en América Latina y el Caribe.

A partir del reconocimiento de una diversidad de procesos de reforma sectorial en la región 1,2, la colaboración IDRC-OPS asignó una importancia fundamental al fortalecimiento de las bases del conocimiento y de la "inteligencia sanitaria” en los países, con el fin de fortalecer la gobernanza e incrementar la equidad de los sistemas de salud. A partir de 1999, en una primera etapa se trabajó de forma conjunta en la revisión y análisis de la investigación sobre reforma del sector salud en la región en el período 1995-2000. La publicación resultante señala que es necesario replantear los patrones predominantes de las reformas en la región, subraya la orientación predominantemente económica de las reformas y su énfasis en aspectos de eficiencia en la gestión de los servicios en desmedro de una reconsideración del modelo de atención, la importancia del rol rector del Estado y la participación social 
en salud, la recuperación de las funciones de salud pública, el acceso universal, la calidad y la equidad en la atención en salud. Por otra parte, fue notoria la ausencia de bases empíricas que informaran las opciones de políticas sobre los procesos de reforma impulsados por actores nacionales e internacionales, como también la baja capacidad de las investigaciones para incidir en la agenda política 3 .

Como parte de este proceso de reflexión y consulta, en el año 2001, IDRC y OPS organizaron un Taller Internacional sobre La Reforma del Sector Salud en las Américas: Fortaleciendo los vínculos entre Investigación y Políticas que tuvo lugar en Montreal, Canadá 4 . Las conclusiones de este taller apuntan a una urgente llamada para la construcción de políticas sustentadas por el mejor conocimiento científico disponible, basadas en procesos decisorios democráticos y participativos, orientadas hacia el logro de la equidad en salud, con énfasis en principios de universalidad y el desarrollo de estrategias que respondan ante los desafíos crecientes de la falta de acceso y condiciones de alta vulnerabilidad de amplios sectores de la población latinoamericana.

\section{Investigación y políticas para la protección social en salud. Construyendo las interfases entre académicos y tomadores de decisión}

Se estima que en América Latina y el Caribe hay más de 200 millones de personas que no tienen asegurada cobertura regular por el sistema/seguro de salud, de los cuales 100 millones aproximadamente no tienen acceso a ningún servicio de salud. Son múltiples los factores relacionados con una exclusión de tal magnitud. La OPS y la Organización Internacional del trabajo (OIT) promueven la superación de esa alta exclusión mediante las estrategias de Extensión de la Protección Social en Salud. Se hace énfasis en la garantía de la sociedad, a través del Estado, de que los individuos y los grupos puedan alcanzar sus necesidades de salud a través de un acceso adecuado a los servicios de salud, independientemente de su capacidad de pago ${ }^{5}$. Para la OPS/Organización Mundial de la Salud (OMS) el esfuerzo de combatir la exclusión en salud es concordante con su mandato de "reducir las iniquidades en acceso a servicios de salud y en su financiamiento" 5 .

IDRC ha dado prioridad al tema de la exclusión en salud a través de su nueva iniciativa programática sobre Gobernanza, Equidad y Salud (GEH). GEH apoya la investigación aplicada que "fortalezca la capacidad de los gobiernos de asegurar la prestación y el financiamiento de servicios de salud y de salud publica equitativos, especialmente para las poblaciones marginadas y sin acceso a servicios" 4

En la actualidad varios países de la región han centrado su atención en la extensión de la protección social en salud ${ }^{4,6}$. Es notoria la persistencia de distintos enfoques y la necesidad de fortalecer las bases científicas y la "inteligencia sanitaria" para la construcción de una visión renovadora de las políticas de salud que asigne una importancia central a la superación de las condiciones predominantes de exclusión e inequidad en el acceso y uso de los servicios de salud 7 . Ello no es posible sin el compromiso de todos, tanto del Estado, como de los tomadores de decisión de nivel nacional y local, académicos, actores sociales y organizaciones de la sociedad civil.

Por otra parte, los modelos tradicionales de producción de nuevos conocimientos en el campo de la investigación en sistemas de salud no han sido capaces de influir en las agendas políticas de los países. Existe una escasa producción científica en este campo y una notoria necesidad de fortalecer las capacidades institucionales.

Esa situación fue explícitamente demostrada por la Iniciativa Salud Pública en las Américas, implementada entre 2000 y 2002 por la OPS y los Estados-Miembros, basada en una intensiva medición del desempeño de las Funciones Esenciales de Salud Pública (FESP) 8. La función de investigación en salud pública (FESP 10) - definida como "la investigación rigurosa dirigida a aumentar el conocimiento que apoye la adopción de decisiones (...), y el desarrollo de soluciones innovadoras en materia de salud publica" 9 - se ha posicionado como el segundo más bajo desempeño promedio regional entre las 11 funciones esenciales de salud pública analizadas, con una distribución bastante homogénea de ese resultado entre los países, y muy pocas excepciones de mejores resultados nacionales 9 .

Con el fin de lograr un impacto en la toma de decisiones, no es suficiente con el diseño de soluciones exclusivamente técnicas. Es necesario construir estrategias de colaboración entre distintos actores y ensayar nuevos puentes de interrelación entre investigación y políticas 10,11. Para ello se requiere lograr una mejor comprensión de los procesos de toma de decisiones en la gestión de los sistemas de salud.

Las consideraciones precedentes han servido como antecedente para la Iniciativa Conjunta sobre Investigación para la Protección Social en Salud en América Latina y el Caribe. La misma apunta a un doble objetivo: (a) promover nuevas estrategias para la extensión de la protección social en salud que respondan a los desafíos de la equidad y acceso universal; e (b) incrementar 
las bases empíricas de las estrategias propuestas, a través de nuevos modelos de interacción entre actores.

La Iniciativa Programática de Gobernanza, Equidad y Salud de IDRC y la Unidad de Políticas y Sistemas de Salud de la OPS/OMS, en colaboración con tres redes sub-regionales de investigación en sistemas y servicios de salud (la Red de Investigación en Sistemas y Servicios de Salud en el Cono Sur, la Red Nevalat para la Región Andina y Centro América, y la Red del Caribe Anglófono para la Investigación en Políticas y Sistemas de Salud), realizaron a comienzos del año 2003 un llamado para la presentación de propuestas sobre el tema de la extensión de la protección social en salud. La convocatoria constituyó la primera fase de la iniciativa IDRC-OPS, a través de la cual se realizó un llamado para apoyar la planificación de nuevos estudios de investigación que estén basados en la generación o consolidación de espacios de diálogo entre tomadores de decisión y académicos. Los equipos postulantes debieron tener una co-responsabilidad en la propuesta lo que implicaba la participación activa de tanto del investigador como del tomador de decisión (pertenecientes al gobierno o a grupos de la sociedad civil). De esta forma se apoyaron nueve equipos que desarrollaron un proceso de interacción para la discusión y el desarrollo de los protocolos de investigación. En una segunda fase, cinco proyectos fueron seleccionados en razón de su relevancia para las políticas de salud regionales y su concordancia con los términos de referencia de la iniciativa, considerando su calidad científica, grado de compromiso y participación de los distintos actores involucrados.

Los fundamentos centrales de esta iniciativa fueron los siguientes:

- Que la formulación de políticas para la Extensión de la Protección Social en Salud requiere de una base de investigación sólida y relevante que puede ser utilizada y complementada con otras fuentes de conocimiento;

- Que esta base de investigación debe ser generada en forma conjunta por los principales actores involucrados, ya sean autoridades de gobierno, o sectores de la sociedad civil;

- Que la mejor forma de involucrar a los usuarios de la investigación es crear mecanismos de participación e interacción a lo largo del proceso de diseño, planificación, ejecución y diseminación de la investigación.

En consecuencia, los mecanismos de financiamiento en ambas fases tuvieron como criterios fundamentales para el otorgamiento de las subvenciones: la evaluación de las características de los equipos, su conformación, el grado de interlocución entre actores académicos y tomadores de decisión, y la participación de otros actores relevantes.

Esta publicación se basa en los resultados del proceso desarrollado por los cinco proyectos seleccionados en la segunda fase, que tiene una duración total de tres años (2004-2006). Estas experiencias, que están siendo desarrolladas en Argentina, Brasil, Colombia (Bogotá y Medellín) y Jamaica, son presentadas en esta revista.

A través de la iniciativa conjunta se espera lograr el desarrollo de una masa crítica significativa de experiencias que integren la investigación con la planificación, ejecución, monitoreo y evaluación de estrategias de extensión de la protección social en salud. En forma simultánea, se ha comenzado a transitar un largo camino para la generación y consolidación de conocimientos y experiencias que analicen y apoyen la construcción de vínculos innovadores entre investigación y políticas en sistemas y servicios de salud. IDRC y OPS esperan a través de este esfuerzo conjunto contribuir y consolidar nuevos espacios de reflexión y acción en salud colectiva en nuestra región. 


\section{Resumen}

La Organización Panamericana de la Salud (OPS) y el Centro Internacional de Investigaciones para el Desarrollo de Canadá (IDRC) han promovido una iniciativa conjunta para el diseño, ejecución y evaluación de estrategias de extensión de la protección social en salud en América Latina y el Caribe. Esta propuesta se basó en una revisión previa de la investigación sobre reformas del sector salud y en las recomendaciones del Taller $\mathrm{La}$ Reforma del Sector Salud en las Américas: Fortaleciendo los Vínculos entre Investigación y Políticas (Montreal, Canadá, 2001). En su primera fase la iniciativa impulsó el desarrollo de propuestas sobre extensión de la protección social en salud que fueron elaboradas en forma conjunta por investigadores y tomadores de decisión. En la segunda fase se apoyó la implementación de cinco de estas propuestas con el propósito de promover el desarrollo de nuevas estrategias de protección social en salud y fomentar nuevos modelos de interacción entre actores. En este número de la revista se analizan los procesos de vinculación entre investigadores y tomadores de decisión en los cinco proyectos apoyados por esta iniciativa.

Política de Salud; Sistemas de Salud; Servicios de Salud

\section{Colaboradores}

Esta nota fue escrita en forma conjunta por todos los autores.

\section{Referencias}

1. Infante A, Mata I, López-Acuña D. Reforma de los sistemas de salud en América Latina y el Caribe: situación y tendencias. Rev Panam Salud Pública 2000; 8:13-20.

2. Vergara C. El contexto de las reformas del sector de la salud. Rev Panam Salud Pública 2000; 8:7-12.

3. Almeida C, Bazzani R, Pittman P, organizadores. Investigación sobre reformas del sector salud en América Latina y el Caribe. Reflexiones sobres sus contribuciones al desarrollo de políticas. Washington DC: Organización Panamericana de la Salud; 2001

4. International Development Research Centre. Health sector reform in the Americas: improving the research to policy interface. http://archive. idrc.ca/lacro/docs/conferencias/foro_documents. html (accedido el 15/Ago/2006).

5. Pan American Health Organization. Extension of social protection in health: joint initiative of the Pan American Health Organization and the International Labour Organization. http:/www.paho. org/English/gov/csp/csp26-12-e.pdf (accedido el 31/Jul/2006).

6. Rosenberg H, Andersson B. Repensar la protección social en salud en América Latina y el Caribe. Rev Panam Salud Pública 2000; 8:118-25.

7. Knaul FM, Arreola-Ornelas H, Mendez O. Financial protection in health: Mexico, 1992 to 2004. Salud Pública Mex 2005; 47:430-9.

8. International Society for Equity in Health. Equity and in Latin America and the Caribbean from 1995 to 2005: approaches and limitations. Toronto: International Society for Equity in Health; 2006

9. Organización Panamericana de la Salud. La salud pública en las Américas. Nuevos conceptos, análisis de desempeño y bases para la acción. Washington DC: Organización Panamericana de la Salud; 2002

10. World Health Organization. Bridging the "knowdo" gap. Meeting on knowledge translation in global health. Geneva: World Health Organization; 2006.

11. International Development Research Centre/Coalition for Global Health Research/Institute of Population Health, University of Ottawa. Knowledge translation in health and development: research to policy strategies. Ottawa: International Development Research Centre/Coalition for Global Health Research/Institute of Population Health, University of Ottawa; 2003.

Recibido el 26/Jul/2006

Aprobado el 31/Jul/2006 